Bull. Austral. Math. Soc.

$65 \mathrm{~J} 15,65 \mathrm{~B} 05,65 \mathrm{~L} 50,65 \mathrm{M} 50$

VOL. 38 (1988) [131-140]

\title{
ON NEWTON'S METHOD AND NONDISCRETE MATHEMATICAL INDUCTION
}

\author{
IOANNIS K. ARgYros
}

\begin{abstract}
The method of nondiscrete mathematical induction is used to find sharp error bounds for Newton's method. We assume only that the operator has Hölder continuous derivatives. In the case when the Fréchet-derivative of the operator satisfies a Lipschitz condition, our results reduce to the ones obtained by Ptak and Potra in $\mathbf{1 9 7 2 .}$
\end{abstract}

\section{INTRODUCTION}

Consider the equation

$$
F(x)=0
$$

where $F$ is a nonlinear operator from a Banach space $E$ into itself. The most important iterative procedure for solving (1) is given by Newton's iteration, namely:

$$
x_{n+1}=x_{n}-\left(F^{\prime}\left(x_{n}\right)\right)^{-1} F\left(x_{n}\right), \quad n=0,1,2, \ldots
$$

One of the basic assumptions for the convergence of (2) is that $F$ must be twicedifferentiable in some ball around the initial iterate (see $[2,6,7])$, or that the linear operator $F^{\prime}$ must satisfy a Lipschitz condition of the form

$$
\left\|F^{\prime}(x)-F^{\prime}(y)\right\| \leqslant l\|x-y\| \quad \text { for all } x, y \in E .
$$

Ptak, among others, has given sufficient conditions for the convergence of (2) to a solution of (1) under assumption (3), using the method of nondiscrete mathematical induction $([3,4])$.

There are, however, many interesting problems in the literature where the operator $F^{\prime}$ does not satisfy $(3),\left([3,5]\right.$ and the references there). Instead $F^{\prime}$ is Hölder continuous on some set $E_{1} \subset E$.

Here, restricting ourselves to operators that are only once differentiable, we extend the results obtained in [4]. When (3) is satisfied, our results reduce to the ones in [4]. In particular, using the method of nondiscrete mathematical induction, as in [4], we study the following important problems:

(i) we give sufficient conditions for the convergence of (2) to a solution $x^{\star}$ of (1);

(ii) we find estimates for the distances $\left\|x_{n}-x^{\star}\right\|, n=1,2, \ldots$

Received 22 October, 1987

Copyright Clearance Centre, Inc. Serial-fee code: 0004-9729/88 \$A2.00+0.00. 
Finally, we provide two examples, one from a real scalar case and one using a second order differential equation, where the results of Ptak, [4] cannot be applied but ours can.

\section{Preliminaries}

We assume that $F$ is once Fréchet-differentiable [2] and $F^{\prime}(x)$ is the first Fréchetderivative at a point $x \in E$. It is well known that $F^{\prime}(x) \in L(E)$, the space of bounded linear operators from $E$ to $E$. We say that the Fréchet-derivative $F^{\prime}(x)$ is Hölder continuous over a domain $E_{1} \subset E$ if for some $c>0, p \in[0,1]$, for all $x, y \in E_{1}$,

$$
\left\|F^{\prime}(x)-F^{\prime}(y)\right\| \leqslant c\|x-y\|^{p} \text {. }
$$

In this case we say that $F^{\prime}(\cdot) \in H_{E_{1}}(c, p)$.

We will need the following result whose proof can be found in [2].

Lemma 1. Let $F: E \rightarrow E$ and $E_{1} \subset E$. Assume $E_{1}$ is open and that $F^{\prime}(\cdot)$ exists at each point of $E_{1}$. If for some convex set $E_{2} \subset E_{1}$, we have $F^{\prime}(\cdot) \in H_{E_{2}}(c, p)$, then for all $x, y \in E_{2}$

$$
\left\|F(x)-F(y)-F^{\prime}(x)(x-y)\right\| \leqslant \frac{c}{1+p}\|x-y\|^{1+p} .
$$

A central part in the method of nondiscrete induction is played by the notion of rate of convergence.

Let $T$ denote the positive real axis or an interval of the form $T=(0, b)$.

Definition 1: A funciton $\omega: T \rightarrow T$ is called a rate of convergence on $T$ if the series

$$
\sigma(r)=\sum_{n=0}^{\infty} \omega^{(n)}(r)
$$

is convergent for each $r \in T$, where the iterates $\omega^{(n)}$ of $\omega$ are defined as follows:

$$
\omega^{(0)}(r)=r, \quad \omega^{(n+1)}(r)=\omega\left(\omega^{(n)}(r)\right), \quad n=0,1,2, \ldots
$$

It can easily be checked that the funcitons $\omega$ and $\sigma$ satisfy the equation

$$
\sigma(\omega(r))=\sigma(r)-r, \quad r \in T
$$

Write $U(x, r)=\{y \in E /\|x-y\|<r\}$. We will need the following lemmas whose proofs can be found in $[4,5]$. 


\section{LEMMA 2.}

(a) If we can attach to the iteration (2) for some $x_{0} \in E$ a rate of convergence $\omega$ on an interval $T$ and a family of sets $Z(r) \subset E, r \in T$ such that:

$$
x_{0} \in Z\left(r_{0}\right) \text { for certain } r_{0} \in T \text {, }
$$

and

$$
(r \in T \text { and } x \in Z(r)) \longrightarrow x-\left(F^{\prime}(x)\right)^{-1} F(x) \in U(x, r) \cap Z(\omega(r)),
$$

then the iteration (2) yields a sequence $\left\{x_{n}\right\} n=1,2, \ldots$ which converges to a point $x^{\star} \in E$, such that:

$$
\begin{gathered}
x_{n} \in Z\left(\omega^{(n)}\left(r_{0}\right)\right), \\
\left\|x_{n}-x_{n-1}\right\| \leqslant \omega^{(n)}\left(r_{0}\right),
\end{gathered}
$$

and

$$
\left\|x_{n}-x^{\star}\right\| \leqslant \sigma\left(\omega^{(n)}\left(r_{0}\right)\right), \quad n=1,2, \ldots
$$

(b) if, in addition, for a certain $n \in\{1,2, \ldots\}$ the condition

$$
x_{n-1} \in Z\left(\left\|x_{n}-x_{n-1}\right\|\right)
$$

if fulfilled, then for this $n$ the inequality

$$
\left\|x_{n}-x^{\star}\right\| \leqslant \beta\left(\left\|x_{n}-x_{n-1}\right\|\right)
$$

is satisfied, where

$$
\beta(r)=\sigma(r)-r
$$

The above Lemma is a corollary of the induction theorem of Ptak [5], which we will use in the above simplified version. As in [4], we call (12) a priori estimates and (14) a psoteriori estimates.

We can now easily prove the following lemma.

LEMMA 3. Let $T$ denote an intcrval of the form $T=(0,1)$. Then the function

$$
\omega(r)=r^{p+1}
$$


is a rate of convergence on $T$ and the corresponding function $\sigma(r)$ satisfies the estimate

$$
0<\sigma(r) \leqslant r+\frac{1}{1-r^{p}} r^{p+1}
$$

\section{Main Results}

Theorem 1. Let $E_{1} \subset E$ and $F: E_{1} \rightarrow E$. Assume $F^{\prime}(\cdot) \in H_{E_{2}}(c, p)$ on a convex set $E_{2} \subset E_{1}$. Let $x_{0}$ be such that:

$$
\left\|F^{\prime}(x)-F^{\prime}\left(x_{0}\right)\right\| \leqslant c_{1}\left\|x-x_{0}\right\|^{p}
$$

for all $x \in E_{2}$ and some $c_{1}>0$.

Assume that $F^{\prime}\left(x_{0}\right)$ has a bounded inverse $F^{\prime}\left(x_{0}\right)^{-1} \in L(E)$ with

$$
\begin{gathered}
\left\|\left(F^{\prime}\left(x_{0}\right)\right)^{-1}\right\| \leqslant d, \\
\left\|\left(F^{\prime}\left(x_{0}\right)\right)^{-1} F\left(x_{0}\right)\right\| \leqslant r_{0},
\end{gathered}
$$

and that the functiong defined by

$$
g(r)=d c_{1} r^{1+p}+\left(\frac{d c r_{0}^{p}}{1+p}-1\right) r-d c_{1} r_{0} r^{p}+r_{0}
$$

has a minimum positive zero $r^{\star}>r_{0}$.

Moreover, assume that

$$
d c_{1}\left(r^{\star}\right)^{p}<1
$$

Then

$$
q=\frac{d c r_{0}^{p}}{(1+p)\left(1-d c_{1}\left(r^{\star}\right)^{p}\right)}<1
$$

If $\vec{U}\left(x_{0}, r^{\star}\right) \subset E_{2}$ then the iteration (2) is well defined, remains in $U\left(x_{0}, r^{\star}\right)$ and converges to a solution $x^{\star}$ of (1).

Proof: By the Banach lemma, $F^{\prime}(x)$ has a bounded inverse, since

$$
\left\|F^{\prime}(x)-F^{\prime}\left(x_{0}\right)\right\| \leqslant c_{1}\left\|x-x_{0}\right\|^{p}<c_{1}\left(r^{*}\right)^{p}<\frac{1}{d}
$$

and

$$
\left\|\left(F^{\prime}(x)\right)^{-1}\right\| \leqslant \frac{d}{1-d c_{1}\left\|x-x_{0}\right\|^{p}}
$$


The operator $P$ given by

$$
P(x)=x-\left(F^{\prime}(x)\right)^{-1} F(x)
$$

is well defined on $U\left(x_{0}, r^{*}\right)$. Assume now that $x, P(x) \in U\left(x_{0}, r^{\star}\right)$ and using (5), we obtain

$$
\begin{aligned}
\left\|P^{2}(x)-P(x)\right\| & =\left\|-\left(F^{\prime}(P(x))\right)^{-1} F(P(x))\right\| \\
& \leqslant \frac{d}{1-d c_{1}\left\|P(x)-x_{0}\right\|^{p}}\left[\left\|F(P(x))-F(x)-F^{\prime}(x)(P(x)-x)\right\|\right] \\
& \leqslant \frac{d}{1-d c_{1}\left\|P(x)-x_{0}\right\|^{p}} \cdot \frac{c}{1+p}\|P(x)-x\|^{1+p} \\
& =\bar{g}\left(\|P(x)-x\|,\left\|P(x)-x_{0}\right\|\right),
\end{aligned}
$$

where

$$
g(v, w)=\frac{1}{1-d c_{1} v^{p}} \cdot \frac{d c w^{1+p}}{1+p} .
$$

Define the real sequence $\left\{s_{k}\right\}, k=0,1,2, \ldots$ by $s_{0}=0, s_{1}=r_{0}$ and

$$
s_{k+1}-s_{k}=\frac{1}{1-d c_{1} s_{k}^{p}} \cdot \frac{d c}{1+p}\left(s_{k}-s_{k-1}\right)^{1+p}
$$

Now we have

$$
\begin{gathered}
s_{2}-s_{1} \leqslant q r_{0}<r_{0} \\
s_{2} \leqslant s_{1}+q r_{0}=r_{0}(1+q)<\frac{r_{0}}{1-q} .
\end{gathered}
$$

Using (23) and $g\left(r^{\star}\right)=0$ we get $r_{0}=(1-q) r^{\star}$. That is

$$
s_{2}<r^{\star} \text {. }
$$

By induction we can easily get

$$
\begin{gathered}
s_{k+1}-s_{k} \leqslant q\left(s_{k}-s_{k-1}\right), \\
s_{k+1}-s_{k}<r_{0},
\end{gathered}
$$

and

$$
s_{k+1}<r^{*}
$$

That is,

$$
\lim _{k \rightarrow \infty} s_{k}=s^{*}, \quad \frac{r_{0}}{1-q}=r^{\star}
$$


By the basic majorant Theorem 2.3 of Rheinboldt [6], there exists an $x^{\star} \in \bar{U}\left(x_{0}, r^{\star}\right)$ such that

$$
P\left(x^{\star}\right)=x^{\star} \quad \text { and } \quad \lim _{k \rightarrow \infty} x_{k}=x^{\star}
$$

Finally,

$$
\begin{aligned}
\left\|F\left(x_{k}\right)\right\| & \leqslant\left\|\left(F^{\prime}\left(x_{k}\right)-F^{\prime}\left(x_{0}\right)\right)\left(x_{k+1}-x_{k}\right)\right\|+\left\|F^{\prime}\left(x_{0}\right)\left(x_{k+1}-x_{k}\right)\right\| \\
& \leqslant\left[c_{1}\left\|x_{k}-x_{0}\right\|^{p}+\left\|F^{\prime}\left(x_{0}\right)\right\|\right]\left\|x_{k+1}-x_{k}\right\| \\
& \leqslant\left(c_{1}\left(r^{\star}\right)^{p}+\left\|F^{\prime}\left(x_{0}\right)\right\|\right)\left\|x_{k+1}-x_{k}\right\|
\end{aligned}
$$

since $x_{k} \in \bar{U}\left(x_{0}, r^{\star}\right)$. Letting $k \rightarrow \infty$, we easily obtain from the above inequality that

$$
F\left(x^{\star}\right)=0
$$

since $\left\{x_{k}\right\}$ is a Cauchy sequence and $\left(c_{1} r^{\star}+\left\|F^{\prime}\left(x_{0}\right)\right\|\right)$ is a constant.

The proof of the theorem is now complete.

Theorem 2. Let $E$ be a Banach space and let $x_{0} \in E$. Let $F$ be an operator mapping $U^{\star}=U\left(x_{0}, r\right)$ into $E$ and suppose that $F(\cdot) \in H_{U^{\star}}(c, p)$. Under the hypotheses of Theorem 1 in $U^{\star}$ assume that for some $r \in T, x_{0}$ and some $p \in[0,1]$ there exists an increasing positive function $h(r)$ such that

$$
\begin{aligned}
h(r) & \geqslant c r^{p}+h(\omega(r)), \\
h(\omega(r)) & \geqslant \frac{c}{p+1}, \quad \omega(r)=r^{p+1} .
\end{aligned}
$$

Then the iteration (2) is well defined, remains in $U^{\star}$ and converges to a solution $x^{\star}$ of (1).

The following statements are true:

$$
\begin{gathered}
x_{n} \in Z\left(\omega^{(n)}\left(r_{0}\right)\right), \\
\left\|x_{n}-x_{n-1}\right\| \leqslant \omega^{(n)}\left(r_{0}\right), \\
\left\|x_{n}-x^{\star}\right\| \leqslant \sigma\left(\omega^{(n)}\left(r_{0}\right)\right)
\end{gathered}
$$

and if

$$
x_{n-1} \in Z\left(\left\|x_{n}-x_{n-1}\right\|\right)
$$

then

$$
\left\|x-x^{\star}\right\| \leqslant \beta\left(\left\|x_{n}-x_{n-1}\right\|\right) .
$$


Here,

$$
\begin{aligned}
Z(r)= & \left\{x \in E /\left\|x-x_{0}\right\| \leqslant \sigma\left(r_{0}\right)-\sigma(r), \quad F^{\prime}(x)\right. \text { has a bounded inverse } \\
& \text { and } \left.\left\|F^{\prime}(x)^{-1}\right\| h(r) \geqslant 1, \quad\left\|\left(F^{\prime}(x)^{-1}\right) F(x)\right\| \leqslant r\right\}, \quad r \in T
\end{aligned}
$$

and where $\omega, \sigma$ and $\beta$ are as defined in Lemma 3.

Proof: By Lemma 2 we need to show that (8), (9) and (13) are satisfied. We easily obtain that $Z\left(r_{0}\right)=\left\{x_{0}\right\}$, so that (7) is satisfied. Let $x \in Z(r)$ and set

$$
y=x-\left(F^{\prime}(x)\right)^{-1} F(x) .
$$

Using (7), (26), (27) and (31), we obtain

$$
\begin{gathered}
\left\|y-x_{0}\right\| \leqslant\|y-x\|+\left\|x-x_{0}\right\| \leqslant r+\sigma\left(r_{0}\right)-\sigma(r)=\sigma\left(r_{0}\right)-\sigma(\omega(r)), \\
\left\|F^{\prime}(y)^{-1}\right\|^{-1} \geqslant\left\|F^{\prime}(x)^{-1}\right\|^{-1}-\left\|F^{\prime}(x)-F^{\prime}(y)\right\| \geqslant h(r)-c r^{P} \geqslant h(\omega(r)) .
\end{gathered}
$$

By (32),

$$
F(y)=F(y)-F(x)-F^{\prime}(x)(x-y)
$$

therefore, (5) and (33) give

$$
\left\|\left(F^{\prime}(y)\right)^{-1} F(y)\right\| \leqslant \frac{1}{h(\omega(r))} \frac{c r^{p+1}}{(p+1)} \leqslant \omega(r),
$$

that is, (9) is satisfied since $y \in Z(\omega(r))$. By Lemma 2 the sequence $x_{n}$ converges to the point $x^{\star}$ and the relations (10)-(12) are satisfied.

The rest of the proof of the theorem follows exactly as in [4].

Set $y=x_{n+1}, x=x_{n}$ in (34) and use the continuity of $F^{\prime}$ to finally obtain $F\left(x^{\star}\right)=0$, that is, $x^{\star}$ is a solution of (1). The proof is now completed using Lemma 3 to obtain the a priori and a posteriori estimates claimed in this theorem.

Note that for $p=1$, Theorem 3.1 in [4] can be obtained as a special case of the above theorem. Remarks similar to the one's following Theorem 3.1 in [4] would follow, but we leave them to the motivated reader. Instead, we provide two possible applications.

Remark. As an example for a function $h$ satisfying (2) and (2 $\tau)$, set

$$
h(r)=c\left(100+r^{p}+2 \ln (r)\right)
$$

where $p=\frac{1}{2}$ and $T=\left(e^{-45}, e^{-1}\right)$. It can easily be checked that (26) and (27) are satisfied. 


\section{ApPlications}

Example 1. Consider the function $G$ defined on $[0, b]$ by

$$
G(t)=\frac{2}{3} t^{3 / 2}+t-3
$$

for some $b>0$.

Let $\|\cdot\|$ denote the max norm on $R$, then

$$
\left\|G^{\prime \prime}(t)\right\|=\max _{t \in[0, b]}\left|\frac{1}{2} t^{-1 / 2}\right|=\infty,
$$

which implies that the basic hypothesis in [2] (or (3)) for the application of Newton's method is not satisfied for finding a solution of the equation

$$
G(t)=\mathbf{0}
$$

However, it can easily be seen that $G^{\prime}(t)$ is Hölder continuous on $[0, b]$ with

$$
c=1 \text { and } \quad p=\frac{1}{2}
$$

Therefore, under the assumptions of Theorem 2, iteration (2) will converge to a solution $t^{\star}$ of (36).

A more interesting nontrivial application for Theorem 2 is given by the following example.

Example 2. Consider the differential equation

$$
\begin{aligned}
x^{\prime \prime}+x^{1+p} & =0, \quad p \in[0,1] \\
x(0) & =x(1)=0 .
\end{aligned}
$$

We devide the interval $[0,1]$ into $n$ subintervals and we set $h=\frac{1}{n}$. Let $\left\{v_{k}\right\}$ be the points of subdivision with

$$
0=v_{0}<v_{1}<\cdots<v_{n}=1 .
$$

A standard approximation for the second derivative is given by

$$
x_{i}^{\prime \prime}=\frac{x_{i-1}-2 x_{i}+x_{i+1}}{h^{2}}, \quad x_{i}=x\left(v_{i}\right), \quad i=1,2, \ldots, n-1 .
$$

Take $x_{0}=x_{n}=0$ and define the operator $F: R^{n-1} \rightarrow R^{n-1}$ by

$$
F(x)=H(x)+h^{2} \varphi(x)
$$


[9]

$$
\begin{gathered}
H=\left[\begin{array}{cccc}
2 & -1 & & \\
-1 & 2 & -1 & 0 \\
& \ddots & \ddots & \ddots \\
0 & -1 & 2 & -1 \\
& & -1 & 2
\end{array}\right], \\
\varphi=\left[\begin{array}{c}
x_{1}^{1+p} \\
x_{2}^{1+p} \\
\vdots \\
x_{n-1}^{1+p}
\end{array}\right]
\end{gathered}
$$

and

$$
x=\left[\begin{array}{c}
x_{1} \\
x_{2} \\
\vdots \\
x_{n-1}
\end{array}\right]
$$

Then

$$
F^{\prime}(x)=H+h^{2}(p+1)\left[\begin{array}{ccc}
x_{1}^{p} & & 0 \\
& x_{2}^{p} & \\
& & \ddots \\
0 & & x_{n-1}^{p}
\end{array}\right]
$$

Newton's method cannot be applied to the equation

$$
F(x)=0 .
$$

We may not be able to evaluate the second Fréchet-derivative since it would involve the evaluation of quantities of the form $x_{i}^{-p}$ and they may not exist.

Let $x \in \mathbf{R}^{n-1}, h \in \mathbf{R}^{n-1} \times \mathbf{R}^{n-1}$ and define the norms of $x$ and $H$ by

$$
\begin{gathered}
\|x\|=\max _{1 \leqslant j \leqslant n-1}\left|x_{j}\right| \\
\|H\|=\max _{1 \leqslant j \leqslant n-1} \sum_{k=1}^{n-1}\left|h_{j k}\right| .
\end{gathered}
$$

For all $x, z \in \mathbf{R}^{n-1}$ for which $\left|x_{i}\right|>0,\left|z_{i}\right|>0, i=1,2, \ldots, n-1$ we obtain, for $p=\frac{1}{2}$ say,

$$
\begin{aligned}
\left\|F^{\prime}(x)-F^{\prime}(z)\right\| & =\left\|\operatorname{diag}\left\{\left(1+\frac{1}{2}\right) h^{2}\left(x_{j}^{1 / 2}-z_{j}^{1 / 2}\right)\right\}\right\| \\
& =\frac{3}{2} h^{2} \max _{1 \leqslant j \leqslant n-1}\left|x_{j}^{1 / 2}-z_{j}^{1 / 2}\right| \leqslant \frac{3}{2} h^{2}\left[\max \mid x_{j}-z_{j} \|^{1 / 2}\right. \\
& =\frac{3}{2} h^{2}\|x-z\|^{1 / 2} .
\end{aligned}
$$


Therefore, under the assumptions of Theorem 2, iteration (2) will converge to the solution $x^{\star}$ of (39).

\section{REFERENCES}

[1] H.T. Davis, Introduction to Nonlinear Differential and Integral Equations (Dover Publ., New York, 1962).

[2] L.V. Kantorovich and G.P. Akilov, Functional Analysis in Normed Spaces (Pergamon Press, Oxford, 1964).

[3] F.A. Potra and V. Ptak, Nondiscrete Induction and Iterative Processes (Pitman Publ., 1984).

[4] F.A. Potra and V. Ptak, 'Sharp error bounds for Newton's Process', Numer. Math. 34 (1980), 63-72.

[5] W.C. Rheinboldt, Numerical Analysis of Parametrized Nonlinear Equations (John Wiley, New York, 1986).

[6] W.C. Rheinboldt, 'A unified convergence theory for a class of iterative processes', SIAM J. Numer. Anal. 5,1 (1968), 42-63.

[7] W.C. Rheinboldt, Iterative Solution of Nonlinear Equations in Several Variables (Academic Press, New York, 1970).

Department of Mathematics, New Mexico State University,

Las Cruces, NM. 88003

United States of America 